\title{
A Comprehensive Analysis of Venture Capital Double Game
}

\author{
Xiong Jixia, Lyu Yanxia \\ School of Health Economics and Management \\ Nanjing University of Chinese Medicine \\ Nanjing, China
}

\author{
Huang Tengfei \\ Nanjing Branch \\ Shanghai Xing Yuan Investment Management Co., Ltd. \\ Nanjing, China
}

\begin{abstract}
This study aims to search for the strategy basis and influencing factors of investors, venture capitalists and entrepreneurs in venture capital, so as to promote the healthy development of the venture capital industry through corresponding institutional arrangements. We make a comprehensive game model between investors, venture capitalists and entrepreneurs to specifically analyze the strategies and game results of them. Investors, venture capitalists and entrepreneurs' strategy choice directly influence the efficiency of venture capital operation and the development of venture capital industry. The study obtains the game solution under different situations, and finds out the main influencing factors as follows: the environmental factor, the capacity of $\mathrm{VC}$ and $\mathrm{VE}$, the moral quality of $\mathrm{VC}$ and $\mathrm{VE}$, the revenue ratio, the share dividend ratio, the supervision strength, the punishment strength and the enforcement strength, the reputation mechanism and so on. We propose changing the value of the major factors through institutional arrangements to optimize game results.
\end{abstract}

Keywords—venture capital; game model; moral hazard

\section{INTRODUCTION}

Venture capital is an emerging financial operation mechanism that professional investors collect funds to provide equity financing to those burgeoning projects or enterprises with enormous potential value and to participate in the management, and ultimately obtain a return on investment through the equity transfer. Development of the venture capital industry has important strategic significance to promote hightech industrialization and optimization of industrial structure, and thus to strengthen the national economy and the comprehensive national power.

The core of developing Venture capital is to find the internal dynamic mechanism of its development and the institutional arrangements to support and enhance the dynamic mechanism, while the point of the internal dynamic mechanism development lies in each Venture participants. Strategies of VC (venture capitalist) and VE (venture entrepreneur) directly affect the operational efficiency of Venture capital, the ultimate income and the development of Venture capital industry. There are two levels of the game in venture capital between investors and $\mathrm{VC}, \mathrm{VC}$ and $\mathrm{VE}$. The two levels of the game are not completely independent. They influence each other mutually. The double game codetermines strategy and utility of investors, $\mathrm{VC}$ and VE, and finally decides operation and development of

This work is supported by Jiangsu Government Scholarship for Overseas Studies and Jiangsu Provincial 333 Project

\section{Venture Capital.}

Sahlman (1994) believed that there was a dual agency relationship between VC, portfolio companies and ultimate investors [1]. There was a problem of moral hazard and adverse selection. As Wright and Robbie (1998) found that a high degree of information asymmetry may be the cause of a lot of very successful businesses later being refused by venture capital, and only a small amount of venture capital investment in early-stage venture [2]. Two-stage surrogate model of Chan et al. (1990) examined the relationship between the skills and control powers of risk entrepreneurs [3]. Cable and Shane (1997) analyzed the cooperative game relationship between VE and VC in the framework of the "prisoner's dilemma" [4].

Zhang Suodi and Hou Jue (2009) analyzed the equilibrium conditions between VC and VE in the venture capital markets with varying degrees of success by complete information dynamic game approach [5]. Tan Xuewei (2013) analyzed the selection process for the mutual $\mathrm{VC}$ and enterprises with the perspective of asymmetric information from previous venture capital investment [6]. Chen Tingqiang (2014) built mathematical model of risk investment and financing contract mechanism design on control transfer and its incentive and restraint of two-stage risk financing, then provided a useful adjustment and adding to the contract mechanism design theory of enterprise's risk financing [7]. Lukas, E (2016) presented a dynamic model of entrepreneurial venture financing under uncertainty based on option exercise games between an entrepreneur and a venture capitalist. They analyzed the impact of multi-staged financing and both economic and technological uncertainty on optimal contracting in the context of $\mathrm{VC}$ financing [8].These highly specialized research provided a good basis for a comprehensive and systematic analysis of venture capital, and provided a reference for the present study.

Because of the serious information asymmetry, moral hazards must be considered [9]. This study attempts to combine the financing and investment game with the moral hazard game to make a comprehensive study of games between investors and VC, VC and VE. The study specifically analyzes strategies of investors, $\mathrm{VC}$ and $\mathrm{VE}$, tries to find out the main factors so as to optimize strategies of $\mathrm{VC}$ and $\mathrm{VE}$ through system designs. We hope to come to the conclusion that can be used to explain development of venture capital and to be the basis of institutional arrangements and innovation so as to promote the healthy development of the venture capital. 


\section{COMPREHENSIVE ANALYSIS OF DOUBLE GAME}

There are two levels of the game in the venture capital operation. First, it is the game between investors and VC. Investors choose whether to invest by examining the capacity and quality of $\mathrm{VC}$ and the expected investment income that they will possess; if they decide to invest, they need to choose the right income allocation programs and monitoring mechanisms; VC choose whether to accept those investments from particular investors and the corresponding levels of endeavor and moral hazard, which has a direct impact on net revenue of venture capital. Second, it is the game between VC and VE. VC choose whether to invest specific venture enterprises by examining the capacity and quality of $\mathrm{VC}$ and the expected investment income that they will possess; if they decide to invest, they need to choose the right allocation programs of stock rights, monitoring mechanisms and the level of value-added services provided by venture enterprises; VE choose whether to accept those investments from VC and the corresponding levels of endeavor and moral hazard behavior; the levels of endeavor and moral hazard behavior of VE as well as the level of value-added services provided by $\mathrm{VC}$ has a direct impact on net revenue. The results of the above double game directly affect the success or failure of the venture capital operations. The more general game results of venture capital decide the condition of the venture capital industry in a country or a region.

\section{A. Assumptions}

1) Investors invest $I$ to $\mathrm{VC}$, entrust $\mathrm{VC}$ with the management and operation of venture capital, and give $\mathrm{VC}$ the incidental revenue ratio $(\alpha)$; venture capital firms invest the risk capital $(I)$ to venture enterprise to obtain equity ratio $(K)$;VE takes in charge of venture enterprise; VC provides value-added services.

2) VC and VE have the incentive to take the self-serving moral hazard behavior, because the high degree of information asymmetry.

3) Investors and VC make the prior game; investors first select the specific incidental revenue ratio as well as the strength of supervision and punishment; VC later selects the levels of endeavor and moral hazard behavior to realize their own utility maximization.

4) VE and VC make the later game; VC first selects the specific percentage of share dividend as well as the strength of supervision and punishment; VE later selects the levels of endeavor and moral hazard behavior to realize their own utility maximization.

\section{B. The expected revenue}

The expected revenue of venture enterprise (R) are codetermined by the capacity $(\theta)$ and endeavor(e) of VC and $\mathrm{VE}$, and it is proportionally shared by the three parties. The three parties make the game mainly based on the implementation and distribution of the expected revenue. The expected revenue will directly affect utility functions as well as the decisions of investors, $\mathrm{VC}$ and $\mathrm{VE}$, so the design of the expected revenue function is very important. The former expected revenue function is the addition function,
$R=e+\theta+\varepsilon$, which has obvious defects. First, it can not reflect the mutual impact of endeavors and capabilities. Second, it ignores the influence of external factors (such as environmental conditions) on the expected benefits. Third, it ignores the impact of the amount invested on the expected return. Fourth, the moral hazard is simplified to reducing endeavors and the negative endeavors, it does not correspond to reality. In reality, endeavors and self-serving moral hazard behavior may exist at the same time. So expected revenue loss $\left(R_{D}\right)$, which may be caused by moral hazard of agents should be considered separately. In addition, the expected revenue of venture enterprises depends on the joint endeavor of $\mathrm{VC}$ and VE. Considering the above factors, the study reconstructs the expected revenue function of venture enterprise.

$$
R=I A e_{1} \theta_{1}+I A e_{2} \theta_{2}+\varepsilon-R_{D 2}
$$

$I$ represent the total investment amount. $e$ represents the endeavor level $(0<e \leq 1), \theta$ represents the capacity $(0<$ $\theta \leq 1)$, Subscript 1 represents VC, and subscript 2 represents VE. $A$ represent the impact of exogenous factors except the capacity and endeavor $(A>0)$, mainly reflecting the environmental factors (policy, law, market sophistication, capital markets, etc.). If the environmental condition is better, $\mathrm{A}$ is bigger. The value of $\mathrm{A}$ in certain period is given. $\varepsilon$ is a random error term (reactive exogenous uncertainties, such as market uncertainty, etc. $)$, and it subjects to $\left(0, \sigma_{\varepsilon}^{2}\right)$ normal.

$R_{D 2}$ indicates the own income caused by the moral hazard behavior of VE; $R_{D 2} \geq 0$; $-\mathrm{R}_{\mathrm{D} 2}$ indicates the expected revenue loss caused by the moral hazard behavior of VE. $R_{D 2}$ can be used to measure the level of the moral hazard behavior.

While venture capital firm gets the revenue of $K R$; VE shares the revenue of $(1-K) R$. Investors and $\mathrm{VC}$ allocate the revenue accordingly. Considering VC may have moral hazard behavior, the revenue in distribution for $\mathrm{VC}$ and investors becomes:

$$
R^{\prime}=K\left(I A e_{1} \theta_{1}+I A e_{2} \theta_{2}+\varepsilon-R_{D 2}\right)-R_{D 1}
$$

$\mathrm{R}_{\mathrm{D} 1}$ represents the own income caused by the moral hazard behavior of $\mathrm{VC}$; $-\mathrm{R}_{\mathrm{D} 1}$ represents the total expected revenue loss caused by the moral hazard behavior of VC. $\mathrm{R}_{\mathrm{D} 1}$ can be used to measure the level of the moral hazard behavior.

Thus, VC shares the revenue of $a R^{\prime}$, and investors share the revenue of $(1-a) R^{\prime}$.

\section{Utility analysis}

On the basis of the expected revenue analysis, we can deduce their respective utilities when investors invest, VC choose to finance and invest, VE choose to finance.

The utility of VE is as follows.

$$
\begin{aligned}
U_{V E} & =W_{2}+(1-K) R-C_{e 2}+R_{D 2}-F_{2} P_{2}-C_{D 2}+H_{2}+S_{2} \\
& =W_{2}+(1-K) R-W_{2} \mathrm{e}_{2}^{2}+R_{D 2}-\mathrm{f}_{2} R_{D 2}\left(\mathrm{p}_{2} \mathrm{C}_{\mathrm{M} 2} R_{D 2}\right) / \mathrm{I}^{2} \\
& -\mathrm{c}_{D 2} \mathrm{R}_{D 2}+h_{2} R+s_{2} R
\end{aligned}
$$


The utility of $\mathrm{VC}$ is as follows.

$$
\begin{aligned}
U_{V C} & =W_{1}+a R^{\prime}-C_{e 1}-a C_{M 2}+R_{D 1}-C_{D 1}-F_{1} P_{1}+a F_{2} P_{2}+h_{1} R^{\prime}+s_{1} R^{\prime} \\
& =W_{1}+a R^{\prime}-C_{e 1}-a C_{M 2}+R_{D 1}-C_{D 1}-\mathrm{f}_{1} R_{D 1}\left(\mathrm{p}_{1} \mathrm{C}_{\mathrm{M} 1} R_{D 1}\right) / \mathrm{I}^{2} \\
& +a \mathrm{f}_{2} R_{D 2}\left(\mathrm{p}_{2} \mathrm{C}_{\mathrm{M} 2} R_{D 2}\right) / \mathrm{I}^{2}+h_{1} R^{\prime}+s_{1} R^{\prime}
\end{aligned}
$$

The utility of investors is as follows.

$$
\begin{aligned}
U_{I} & =(1-a) R^{\prime}-W_{1}-C_{M 1}-(1-a) C_{M 2}+F_{1} P_{1}+(1-a) F_{2} P_{2} \\
& =(1-a) R^{\prime}-W_{1}-C_{M 1}-(1-a) C_{M 2}+\mathrm{f}_{1} R_{D 1}\left(\mathrm{p}_{1} \mathrm{C}_{\mathrm{M} 1} R_{D 1}\right) / \mathrm{I}^{2} \\
& +(1-a) \mathrm{f}_{2} R_{D 2}\left(\mathrm{p}_{2} \mathrm{C}_{\mathrm{M} 2} R_{D 2}\right) / \mathrm{I}^{2}
\end{aligned}
$$

In the above formulas, there are some variables with same meaning. In fact, subscript 1 represents VC, and subscript 2 represents VE. W represents the fixed income. $C_{e}$ represents the endeavor cost. Generally, the endeavor cost incrementally increases as the level of endeavor increases, so $C_{e}{ }^{\prime}>0, C_{e}{ }^{\prime \prime}>0$. Theoretically the fixed income is paid for efforts, so the fixed income should be paid based on the estimated cost of efforts. This article assumes that the fixed income is paid for the cost at $100 \%$ effort. So we construct the endeavor cost function, $C_{e}=W e^{2}, e=C_{e}{ }^{\prime} / 2 W$, which conforms to the regularity that the endeavor cost incrementally increases as the level of endeavor increases. $C_{M}$ is the monitoring costs, it can reduce the level of the moral hazard behavior. $F$ is not only the penalty for the moral hazard behavior, but also the compensation for the principal. $\mathrm{F}$ is positively correlated to $\mathrm{R}_{\mathrm{D}}$ (the income of moral hazard behavior). Assuming $F=f R_{D} ; f$ is the punishment multiples; $f>1 . P$ is the probability of finding the moral hazard behavior, it is positively correlated to $C_{M} / I$, and $R_{D} / I$. Thus, we design the function, $P=p C_{M} R_{D} / I . p$ is the probability coefficient of finding the moral hazard behavior, which is determined by the capacity of the principal, the information availability and so on. $C_{D}$ represents the psychological cost of the moral hazard behavior, which is positively correlated to $R_{D}$. Assuming $C_{D}=c_{D} R_{D .} c_{D}$ is the psychological cost coefficient of the moral hazard behavior, and it is determined by their moral qualities and cultural backgrounds. $S$ is the self-realization utility, $s$ is the coefficient of the self-realization utility, which is determined by their own qualities and cultural backgrounds. $0<s_{s}<1 . S_{l}$ is positively correlated to the earnings of venture capital, $S_{1}=s_{1} R^{\prime} . S_{2}$ is positively correlated to the earnings of venture enterprise, $S_{2}=s_{2} R$. H is the reputation utility, $\mathrm{h}$ is the coefficient of the reputation utility, $0<h<1$. The more the follow-up games are, the more perfect the social reputation mechanism is, and the greater $\mathrm{h}$ is. $\mathrm{H}_{1}$ is positively correlated to the earnings of venture capital, $H_{l}=h_{1} R^{\prime} . H_{2}$ is positively correlated to the earnings of venture enterprises, $H_{2}=h_{2} R$.

\section{The choice of the endeavor level and the moral hazard level of $\mathrm{VC}$}

Firstly, we analyze the choice of the endeavor level of VC. According to the first order optimum condition of $d U_{V C} / d e_{1}=0$, the optimal endeavor level of $\mathrm{VC}$ is as follows.

$$
e_{1}=I A \theta_{1}\left(a K+h_{1}+s_{1}\right) / 2 W_{1}
$$

Secondly, we analyze the choice of the moral hazard behavior level of VC. According to the first order optimum condition of $d U_{V C} / d R_{D 1}=0$, the optimal moral hazard behavior level of $\mathrm{VC}$ is as follows.

$$
R_{D 1}=I^{2}\left(1-c_{D 1}-a K-s_{1}-h_{1}\right) / 2 f_{1} p_{1} C_{M 1}
$$

When $1-c_{D 1}-a K-s_{1}-h_{1} \leq 0$, VC won't have moral hazard behavior. It means they will choose the strategy of no moral hazard behavior. However, there is a special case of some moral hazard behavior in the strategy of no moral hazard behavior. That is when $e_{1}=\operatorname{IA} \theta_{1}\left(a K+h_{1}+s_{1}\right) / 2 W_{1}, R_{D 1}=0$. But in reality, the condition of $1-c_{D 1}-a K-s_{1}-h_{1} \leq 0$ is difficult to meet, VC usually choose the strategy of having some moral hazard behavior. We can reduce the moral hazard level and enhance the endeavor level through system designs in order to optimize the outcome of the game.

\section{E. The choice of the endeavor level and the moral hazard level of $V E$}

Firstly, we analyze the choice of the endeavor level and the moral hazard behavior level when VE take the strategy of some moral hazard behavior.

VE choose the endeavor level in accordance with the principle of their own utility maximization. According to the first order optimum condition of $d U_{V E} / d e_{2}=0$, we have:

$$
e_{2}=I A \theta_{2}\left(1-K+h_{2}+s_{2}\right) / 2 W_{2}
$$

VE choose the moral hazard level in accordance with the principle of their own utility maximization. According to the first order optimum condition of $d U_{V E} / d R_{D 2}=0$, we have:

$$
R_{D 2}=I^{2}\left(K-c_{D 2}-s_{2}-h_{2}\right) / 2 f_{2} p_{2} C_{M 2}
$$

When $K-c_{D 2}-s_{2}-h_{2} \leq 0$, VE won't have moral hazard behavior. It means they will choose the strategy of no moral hazard behavior. However, there is a special case of some moral hazard behavior in the strategy of no moral hazard behavior. That is when $e_{2}=\operatorname{IA} \theta_{2}\left(1-K+h_{2}+s_{2}\right) / 2 W_{2}$, $R_{D 2}=0$. But in reality, the condition of $K-c_{D 2}-s_{2}-h_{2} \leq 0$ is difficult to meet.

If the utility of complete moral hazard behavior is greater than the utility of incomplete moral hazard behavior, VE will select the complete moral hazard behavior, and then there is no selection of the endeavor level.

\section{F. Results and discussion of the game}

According to the principles of economics, the optimal choices are the realistic choices of rational people. Based on the foregoing analysis, $\mathrm{VC}$ will be in accordance with the principle of their own utility maximization to choose the endeavor level and the moral hazard level according to equation (6) and (7); VE will be in accordance with the principle of their own utility maximization to choose the endeavor level and the moral hazard level according to equation (8) and (9). 
These four variables are substituted into the expected revenue function to find the expected revenue in this case, and they can also be substituted into the utility functions of investors and $\mathrm{VC}$ to find their own utility values for strategy selections. Investors and $\mathrm{VC}$ have invested in this game, so they can only adjust the next round of investment and financing strategies accordingly. Therefore, in the case that rules of the game are established, the strategy of $\mathrm{VC}$ is established. They accept investments from investors, invest to $\mathrm{VE}$, and be in accordance with the principle of their own utility maximization to choose the endeavor level and the moral hazard level according to equation (6) and (7).When $1-c_{D 1}-a K-s_{1}-h_{1} \leq 0$, VC choose the strategy of no moral hazard behavior; $R_{D 1}=0$, the endeavor level is invariant.

For VE, their strategy options include the strategy of no moral hazard behavior, the strategy of some moral hazard behavior and the strategy of complete moral hazard behavior. VE will next compare their own utilities of three strategies and finally choose the strategy which makes their own utility maximization.

The utility of VE without moral hazard behavior is as follows.

$$
U_{V E 1}=W_{2}+(1-K) R_{1}-C_{e 2}+h_{2} R_{1}+s_{2} R_{1}
$$

The utility of VE with some moral hazard behavior is as follows.

$$
\begin{aligned}
U_{V E 2}= & W_{2}+(1-K)\left(R_{1}-R_{D 2}\right)-C_{e 2}+R_{D 2}-F_{2} P_{2} \\
& -C_{D 2}+h_{2}\left(R_{1}-R_{D 2}\right)+s_{2}\left(R_{1}-R_{D 2}\right)
\end{aligned}
$$

The utility of VE with complete moral hazard behavior is as follows.

$$
U_{V E 3}=I_{1}-F_{3} P_{3}-c_{D 2} I_{1}-h_{2} I_{1}-s_{2} I_{1}
$$

$\mathrm{F}_{3}$ represents the punishment that VE suffered for complete moral hazard behavior. $\mathrm{P}_{3}$ is the probability that VE suffered punishments for complete moral hazard behavior.

If the utility of VE with some moral hazard behavior is the largest, VE will choose the strategy of some moral hazard behavior as follows.

$$
\begin{array}{r}
e_{2}=I A \theta_{2}\left(1-K+h_{2}+s_{2}\right) / 2 W_{2} \\
R_{D 2}=I^{2}\left(K-c_{D 2}-s_{2}-h_{2}\right) / 2 f_{2} p_{2} C_{M 2}
\end{array}
$$

This situation is the most common one in reality. We can reduce the moral hazard level and enhance the endeavor level through system designs to make them choose the strategy of the high endeavor and the low moral hazard in order to increase venture capitals earnings and promote the development of the venture capital industry.

If $K-c_{D 2}-s_{2}-h_{2} \leq 0$, the utility of VE without moral hazard behavior is the largest, VE will choose the strategy of no moral hazard behavior as follows.

$$
e_{2}=I A \theta_{2}\left(1-K+h_{2}+s_{2}\right) / 2 W_{2}, R_{D 2}=0
$$

This requires a high level of spiritual qualities of VE and a very sound social reputation mechanism at the same time, which are difficult to achieve in reality.

If the utility of VE with complete moral hazard behavior is the largest, VE will choose the strategy of complete moral hazard behavior. Investors and VC will suffer huge losses, which will have seriously negative impacts on the venture capital industry and leads to the next round of investors and VC not dare to reinvest. If it is generally that VE have complete moral hazard behavior, investors will not invest and the venture capital industry will decline. So we must avoid the emergence of this result through system designs.

\section{CONCLUSIONS AND DISCUSSIONS}

1) The strategy selections of VC and VE directly determine the benefits of venture capital, and the results of the double game directly affect the success or failure of the venture capital operations. The more common game results of venture capital operations in a country or a region decide the development of the venture capital industry. We can optimal game results and promote the healthy development of the venture capital industry through making institutional arrangements to change the utility from different strategies of investors, VC and VE.

2) The important factors that affect the game results of venture capital. The environmental factor (A) (policy and market environment), the capacity of $\mathrm{VC}$ and $\mathrm{VE}(\theta)$, the moral quality of $\mathrm{VC}$ and $\mathrm{VE}$ (deciding the value of $\mathrm{cD} 、 \mathrm{~s}$ ), the incidental revenue ratio (a), the share dividend ratio of venture-capital firms $(\mathrm{K})$, the supervision strength $(\mathrm{CM} / \mathrm{I})$, the punishment strength (f) and the enforcement strength for moral hazard behavior, the monitoring capacity and the information availability which deciding the probability coefficient of moral hazard behavior (p), the reputation mechanism (determining the value of $h$ ) and so on. Among the influencing factors, the incidental revenue ratio of VC (a), the share dividend ratio of venture capital firms $(\mathrm{K})$, the punishment strength for moral hazard behavior (f), the monitoring costs $(\mathrm{CM})$ and so on are factors about arrangements of specific operating mechanism of venture capital. Each gamer will constantly adjust to improve the utility. And other factors that affect the game results are mostly related to the environment. For example, good policy and market environment can increase the value of $\mathrm{A}$, improve the expected revenue, and then optimize the game results. The moral qualities of venture capitalists and entrepreneurs are associated with the cultural environment. A good cultural environment can increase the value of $\mathrm{cD}$ and $\mathrm{s}$, it helps to optimize the game result.

3) We can analyze the game results through the factors that affect the game results, explain the operation and development status of venture capital, and hereby improve certain factors through corresponding arrangements so as to perfect the strategy selection of each participant, make game results generally develop towards the better ones for societ 
and promote the healthy development of the venture capital industry. As many influencing factors codetermine the game result, it can not be confined to a single factor in an analysis. All the factors must be taken into account. When we try to optimize the game results through institutional arrangements, attention should be paid to the systemic and coordination. The venture capital industry can achieve good development by improving internal operating mechanism of venture capital (mainly institutional arrangements of venture-capital organizations and venture enterprises) together with the external environment.

4) Environment plays an important role in the venture capital game. The government has the responsibility to provide good policy and legal environment to promote the development of venture capital. It is documented that public market signals affect venture capital investing [10]. Regulating and improving the public market is very important.In addition, the impact of culture environment on venture capital can not be ignored. Culture affects gamers' utility function, thus influences the behavior choices of gamers, and ultimately affects the game result. Suitable culture is a key factor to the sustained and stable development of venture capital. Therefore, we must strengthen institutional construction of venture capital and the corresponding culture as well.

\section{ACKNOWLEDGMENT}

We would like to thank the support of the key discipline of Nanjing University of Chinese Medicine.

\section{REFERENCES}

[1] Sahlman W A, "Insights from the Venture Capital Model of Project Governance," Business Economics, vol. 29, pp. 35-48, March 1994.

[2] Wright, M. and Robbie, K, "Venture Capital and Private Equity: a Review and Synthesis," Journal of Business Finance and Accounting, vol. 25, pp. 521-563,May 1998.

[3] Chan Y, Siegel D. Thakor A Leaning, "Corporate Control and Performance Requirements in Venture Capital Contracts," International Economic Review, vol. 31, pp.393-414, 1990.

[4] Daniel M. Cable and Scott Shane. "A Prisoner's Dilemma Approach to Entrepreneur-Venture Capitalist Relationships,"The Academy of Management Review, vol. 22, pp. 142-176 ,Jan., 1997

[5] Zhang Suodi, Hou Jue, Bai Yuanping, "Complete Information Dynamic Game Analysis on Venture Capital Market," China Market, pp. 67-69, 75, July 2009.

[6] Tanxue Wei, "Venture Capitalists and Venture Enterprises - Game Analysis under Information Asymmetry," Technology and Market, vol. 20, pp. 126-127, March 2013.

[7] Chen Tingqiang; Ding Shaohua; He Jianmin; Li Xindan. "Research on control right transfer and incentive mechanism in risk enterprise financing", Systems Engineering-Theory \& Practice, vol.34, pp. 11451152,May 2014.

[8] Lukas, E; Molls, S; Welling, A," Venture capital, staged financing and optimal funding policies under uncertainty" ,European Journal of Operational Research, vol.250, pp.305-313,Apr, 2016

[9] Cao Guohua, Geng Chaogang, "Game Analysis of Decision-making on Venture Investment Institutions under Competition Mechanism," Journal of Systems Engineering, vol. 24, pp. 596-601, May 2009.

[10] Gompers P, Anna Kovner, Josh Lerner and David, Scharfstein, "Venture Capital Investment Cycles: the Impact of Public Markets," NBER, Working Paper 11385, May 2005. 